\title{
Construcción neoliberal de la política social chilena en el discurso de Pinochet
}

\author{
Juan Saavedra Vásquez \\ Universidad del Bío-Bío, Concepción, Chile
}

\author{
Fernando Farías Olavarría \\ Universidad del Bío-Bío, Concepción, Chile
}

\section{Construcción neoliberal de la política social chilena en el discurso de Pinochet}

Resumen: Los mensajes presidenciales o Cuentas Públicas Anuales de los presidentes de la República do Chile constituyen una tradición en la que se declara el contenido de las políticas públicas en un próximo periodo anual. Atendiendo la actualidad de su contenido, a través de un estudio descriptivo-interpretativo basado en minería de textos y análisis de correspondencias, se observaron los discursos presidenciales realizado por el dictador Augusto Pinochet durante los años 1981 y 1989 atendiendo su carácter neoliberal. El análisis permite establecer las bases neoliberales de la política social de la dictadura, en ámbitos relevantes: ideológico, mercadoeconómico, institucional y social propiamente tal.

Palabras clave: Neoliberalismo. Discurso. Dictadura. Política social.

\section{Construção neoliberal da política social chilena no discurso de Pinochet}

Resumo: As mensagens presidenciais ou Contas Públicas Anuais dos presidentes da República de Chile constituem uma tradição na qual se declara o conteúdo das políticas públicas em um próximo período anual. Atendendo a atualidade de seu conteúdo, a través de um estudo descritivo-interpretativo baseado na investigação de textos e análises de correspondências, foram observados os discursos presidenciais realizados pelo ditador Augusto Pinochet desde 1981 até 1989 e atendendo seu caráter neoliberal. A análise permite estabelecer as bases neoliberais da política social da ditadura em âmbitos relevantes: ideológico, mercado-econômico, institucional e social propriamente dito.

Palavras-chave: Neoliberalismo. Discurso. Ditadura. Política social.

\section{Neolibereal Construction of Chilean Social Policy in the Discourse of Pinochet}

Abstract: The Annual Public Account issued by the presidents of the Republic of Chile are a tradition in which the president presents the public policies for the coming year. Recognizing the timeliness of their content, this is a descriptive-interpretive study based on an analysis of texts and correspondence related to the presidential discourses issued by the dictator Augusto Pinochet from 1981-1989 and their neoliberal character. The analysis allows establishing the neoliberal base of the social policy of the dictatorship, in relevant realms: ideological, market-economic, institutional and social.

Keywords: Neoliberalism. Discourse. Dictatorship. Social Policy. 


\section{Introducción}

Uno de los desafíos del Trabajo Social es atender a los contextos históricos y políticos que sostienen las diversas formas de intervención social, sobre todo, en sus implicancias sobre las prácticas profesionales resultantes. Este artículo se enmarca en el proyecto de investigación UBB 131324 2/R titulado "Discursos y prácticas de intervención situada", en el cual se plantea la pregunta sobre los discursos políticos de la intervención social. Dada la relevancia fragmentaria de la Dictadura Militar de Augusto Pinochet en Chile, resulta de interés aproximarnos a los contenidos de los discursos a través del análisis de correspondencias lexicales de los mensajes presidenciales pronunciados los días 11 de septiembre (aniversario del Golpe de Estado) entre los años 1981 y 1989, de modo tal, poder contar con una panorámica de los principales rasgos neoliberales de la política social de Pinochet en los ámbitos ideológico, mercado-económico, institucional y social. Los resultados investigativos facilitan la visualización del discurso como un aspecto central en la configuración de la intervención social contemporánea.

\section{Los discursos presidenciales en Chile: comunicación y discurso político}

Los Mensajes Presidenciales o Cuentas Públicas Anuales de los presidentes de la república constituyen una tradición de origen británica, establecida por primera vez en Chile en la Constitución de 1833. En la Carta Constitucional de 1925 se fija la obligación del presidente de la república de rendir cuenta ante el Congreso todos los 21 de mayo, fecha que daba inicio al periodo ordinario de sesiones en el Parlamento y que concluía los 18 de septiembre de cada año, práctica realizada continuamente, salvo durante la dictadura de Carlos Ibáñez en la década de 1930. La tradición es interrumpida el año 1973, con el advenimiento de la dictadura militar encabezada por el general Augusto Pinochet, pero retomada en 1981, en el aniversario anual del Golpe de Estado (cada 11 de septiembre). A partir de la restauración del régimen democrático en 1990, nuevamente la Cuenta Pública es desarrollada los días 21 de mayo en la apertura de las sesiones ordinarias del Congreso Nacional.

Se trata de un mensaje público, en el cual el Presidente de la República hace un balance de los aspectos centrales de la gestión gubernamental ejercida durante el periodo anterior, también constituye la oportunidad para declarar los lineamientos más relevantes de la política social para el periodo anual que sucede.

De acuerdo a Gerstlé (2005), la comunicación políti-

\section{Los resultados investigativos}

facilitan la visualización del

discurso como un aspecto

central en la configuración de

la intervención social

contemporánea. ca es una vía alternativa a la resolución violenta del conflicto por el poder. En el marco de la comunicación entre gobernantes y gobernados, el discurso político es clave para entender los aspectos prácticos, éticos y volitivos de la acción de los gobiernos. Este aspecto es relevante en el ámbito de las ciencias sociales y las humanidades, tal como lo evidencian, entre otros, las posiciones de Van Dijk (1992, 2003, 2007), Larraín (2010), Fairclough (1995, 2003), Wodak (2003), Sollon (2003), entre otros múltiples autores. Desde una óptica crítica, Verón (1987, p. 13) señala que el discurso político es uno de los primeros objetos del análisis discursivo, de modo tal que es posible articular este discurso "a la producción discursiva explícitamente articulada a las instituciones del Estado".

Los discursos políticos están conformados por enunciaciones que constituyen afirmaciones cargadas de historicidad, al estar de ancladas a sujetos, situaciones y escenarios en un momento determinado. En este marco, Verón (1987) explica que en la relación al enunciador, existes tres tipologías de destinatarios que dan respuestas a la lucha entre distintos enunciadores colocados en condición de adversidad. Así señala que: a) el tipo "prodestinatario" emerge cuando el discurso está dirigido al partidario, aquel que participa de las mismas ideas del enunciador, adhiriendo al marco valórico y a los objetivos políticos. Por su parte, b) el "contradestinatario" es el adversario, en el cual hay una inversión del supuesto-creencia del enunciador, esto es, que para estos destinatarios el enunciador operaría desde desconfianza hacia el público. Por tanto, es objeto de la exclusión discursiva o del enfrentamiento. Pero, nos dice Verón (1987, p. 14), c) el análisis del discurso político revela la presencia de un "paradestinatario", que representa a los sectores de la ciudadanía "que se mantienen, en cierto modo, fuera del juego, y que en los procesos electorales, son identificados habitualmente como "los indecisos"; si votan, deciden su voto a último momento". 
De la lectura de Verón se entiende que el enunciador del discurso político opera desde ciertos sentidos o "componentes", cuya intensidad ira variando en función de los contextos o circunstancias del destinatario de la comunicación. De esta forma, el "componente descriptivo" evidencia la constatación o balance de hechos generalmente dirigidos al contradestinatario para demostrar logros y contrastes. El componente "didáctico" parte de la formulación de la "verdad universal", sin temporalidad y con sujetos difusos, en la que busca instalar ciertos contenidos en los sujetos destinatarios. Por su parte, el componente "prescriptivo", comunica el deber ser, los imperativos universalizables, generalmente declarados en forma impersonal (paradojalmente esta distancia es legitimadora del enunciador). Estos enunciados prescriptivos están genéricamente dirigidos a los paradestinatarios y prodestinatarios. Por último, el componente "programático" es aquel de las promesas, anuncios y compromisos, de lo que se "puede hacer". Es predominantemente estructurado en tiempo futuro, con abundante uso de nominalizaciones (Ejm. "Chile quiere avanzar..."). Dirigido principalmente a partidarios y prodestinatarios, también refiere por oposición a los contradestinatarios, por ejemplo, al justificar los anuncios en lo que no fue realizado.

Estas comunicaciones no sólo están circunscritas a textos, declaraciones públicas o apariciones de las comunicaciones en los medios de comunicación, sino que obedecen a rituales tradicionales establecidas en los diversos países. En el caso de Chile, la tradición de comunicación más importante es la del mensaje presidencial o cuenta pública a la nación, instaurada desde 1926, y que incluso fue observada en los años del terror dictatorial (1973-1989), aun cuando, en la lógica de refundación nacional de la dictadura de Pinochet, simbólicamente el día elegido para este mensaje es el 11 de septiembre, aniversario del golpe de Estado que derribó al gobierno constitucional del presidente Salvador Allende.

\section{Dictadura militar y neoliberalismo}

Conocido internacionalmente, el régimen genocida de Augusto Pinochet accede al poder en septiembre de 1973 por una conjura militar alentada por los sectores conservadores chilenos y enmarcada en los juegos geoestratégicos de las potencias mundiales de la época. Interesado primero en exterminar a militantes de izquierda y cualquier foco de potencial insurrección al nuevo orden, el régimen militar pronto requiere de un modelo social-económico para reestructurar el país. Carballo et al. (1990) señalan que no es sino hasta 1974 que la influencia de la Escuela de Chicago se hace notar tanto en la economía como en el aparato público chileno, en una "innovadora" perspectiva que será posteriormente denominada como neoliberalismo, cuya presencia se ha extendido mundialmente en los últimos treinta años.

El liberalismo es entendido en general como una corriente de pensamiento social-político que toma su forma moderna a partir de los siglos 17 y 18, en su propósito de oponerse al despotismo y las monarquías absolutas de Europa. Aun cuando recibe influencias de la ilustración (principalmente de Voltaire y Rousseau), el reconocimiento del liberalismo como entidad política no es evidente sino hasta mediado del siglo 19, cuando surge el Partido Liberal Inglés. Es en este marco que se produce la génesis más profunda de la revolución burguesa cuyas consecuencias son observables hasta nuestros días. A este respecto, Foucault señala que liberalismo no quiere decir más libertad para los sujetos. Primero, porque el traspaso de la monarquía administrativa europea a regímenes republicanos no conlleva cambios en los mecanismos de gestión del aparato burocrático. En segundo lugar, porque el uso del término "liberal" no quiere decir que la gubernamentalidad deje más espacios para la libertad. El uso de la palabra libertad en este contexto está reservado para el mercado, y en este sentido, la nueva razón gubernamental es consumidora-productora de libertad. "El liberalismo se plantea simplemente lo siguiente: voy a producir para ti lo que se requiere para que seas libre" (FOUCAULT, 2012, p. 84). Si en este régimen la libertad es algo que se fabrica, el principio de cálculo de dicha producción está dado por la seguridad.

Según Elster (1989), hasta mediados del siglo 19 - tiempos contemporáneos a Marx - los capitalistas no querían tener participación en el poder político, pues implicaba que los dos enemigos de la clase trabajadora el capital y el gobierno - se unieran en una sola entidad, trayendo como efecto una alta inestabilidad social. Tímidamente esta idea comienza a revertirse durante las décadas siguientes, hasta que pasada la influencia del new deal de la presidencia de Roosevelt en Estados Unidos, y los resultados de la II Guerra Mundial, influirán decididamente a la aparición de un nuevo discurso sobre la libertad económica, la relación Mercado-Estado y el debilitamiento del Estado de Bienestar, enmarcado bajo la llamada "Escuela de Chicago".

La dictadura de Pinochet (1973-1990) siguió con especial vehemencia estas premisas del neoliberalismo. Los primeros años del régimen buscaron asentar el poder, destruir la disidencia y retrotraer algunas de las conquistas de los sectores populares, no obstante que la más estratégica de todas - la nacionalización de la industria cuprífera - seguiría en manos del Estado hasta nuestros días. Para el año 1980, Pinochet somete un proyecto Constitucional a consulta de la ciudadanía en un plebiscito irregular, en el cual se aprueba el nuevo 
texto constitucional. A partir de 1981 y hasta el fin formal de la dictadura, el régimen se dedica a la tarea de profundizar la revolución neoliberal, atacando el núcleo central del sistema de políticas sociales asistenciales (ILLANES, 2010) organizado desde la década de 1920, y que se plasmó concretamente durante décadas en las áreas de salud, educación, vivienda y previsión.

$\mathrm{Al}$ analizar el contenido del discurso que instaura el neoliberalismo en Chile se busca comprender de mejor forma los contextos de la intervención social, a partir de la vigencia de la Constitución de 1980, y cuya expresión se evidencia centralmente en los mensajes presidenciales del dictador Augusto Pinochet entre los años 1981 y 1989.De esta forma, se pretende establecer una base de discusión sobre la continuidad de dicha impronta neoliberal y, en un posterior estudio, abordar las continuidades discursivas del neoliberalismo en la contemporaneidad de la acción político-social chilena.

\section{Material y métodos}

Se trata de un estudio descriptivo-interpretativo, bajo un diseño longitudinal retrospectivo. La unidad de análisis está dada por la producción de los discursos presidenciales realizado por el dictador, durante los años 1981 y 1989, los cuales están disponible en los archivos digitalizados de la Biblioteca Nacional de Chile. A partir de los discursos presidenciales se construyó el corpus de análisis, específicamente lo referido a la construcción de las políticas sociales, vinculadas a los temas como "salud", "educación", "vivienda", "trabajo" e "ingresos", así como, a la contextualización de éstas, lo cual estaba anclada en la construcción de la nueva institucionalidad que se venía gestando en Chile, el neoliberalismo.

Para el análisis de corpus se procedió a través de las herramientas de la minería de textos, específicamente lematización y análisis de correspondencias de los mensajes presidenciales de Pinochet en el periodo estudiado, para lo cual se utilizó el software $T$-Lab 8.1. Este procedimiento permite reducir la complejidad del corpus a través de una serie de algoritmos estadísticos que permiten alcanzar, a la vez, una panorámica concisa pero global del texto. Sin embargo, para cumplir con los objetivos de este artículo, se detallarán sólo los resultados del análisis de las correspondencias lexicales.

\section{Análisis y resultados}

El análisis de las correspondencias lexicales es una técnica del análisis factorial que permite extraer las nuevas variables, los factores, así como resumir la información relevante contenida en el corpus textual. Cada factor se fija en una dimensión espacial - que se puede representar como un eje factorial - cuyo centro es el valor "0", y que se desarrolla hacia el fin negativo y positivo de manera bipolar. De este modo, los lemas que se ubican en los polos opuestos tendrán entre ellos la máxima diferencia.

El plano factorial presenta, en el primer factor (eje X) la información en el continuo que avanza desde la política en el sentido de la institucionalidad-ideológica fundante, hacia la política como gestión de los programas sociales. En la polaridad negativa sitúa los lemas vinculado a la construcción de la nueva institucionalidad junto a su justificación ideológica, en donde, se encuentra la construcción discursiva paradestinatarios, prodestinatarios (valores, Constitución, político, institucionalidad, Chile, libertad, nación, chileno, pueblo, electoral, poder ejecutivo, homenaje, orden) y contradestinatarios (soviético, comunismo, violencia, político, totalitario, lucha).

En la polaridad positiva del mismo eje, da cuenta de la política como Gestión de Programas Sociales, en donde, se aprecia los siguientes lemas con huella semántica (salud, educación, vivienda, trámite, rural, urbano, mejoramiento, infantil, obras, programas, mortalidad, nacidos, cobertura, incrementar, subsidio, recurso, millones).

El segundo factor (eje Y) estructura la información desde el continuo de la política social sobre los individuos (micro) hacia la política social sobre el colectivo (macro). Esta lectura permite sostener que el discurso de la dictadura genera rasgos biopolíticos, según lo expresado por Foucault (2012). Los lemas vinculados a esta relación son: vivos, nacidos, mortalidad, niños. En cambio, los lemas asociados a las políticas sociales de orden macro son: los programas sociales de los sectores vivienda, salud, educación, trabajo.

En cuanto a los cuadrantes, estos ordenan el discurso de la dictadura en materia de política social en cuatro sectores, a saber, el cuadrante I (+-) agrupa los discursos vinculado a obras e implementación de la política social, dirigido principalmente a los prodestinatarios y paradestinatarios; el cuadrante II (-+) dirigido también a paradestinatarios y prodestinatarios, en lo referente a la construcción de la nueva institucionalidad neoliberal en materia de política social; el cuadrante III (-) indica lo político ideológico destinado principalmente a los contradestinatarios y finalmente el IV cuadrante (++), agrupa los lemas discursivos a la implementación (logros y proyectos) de las políticas sociales. 


\section{Figura 1: Análisis de correspondencias lexicales}

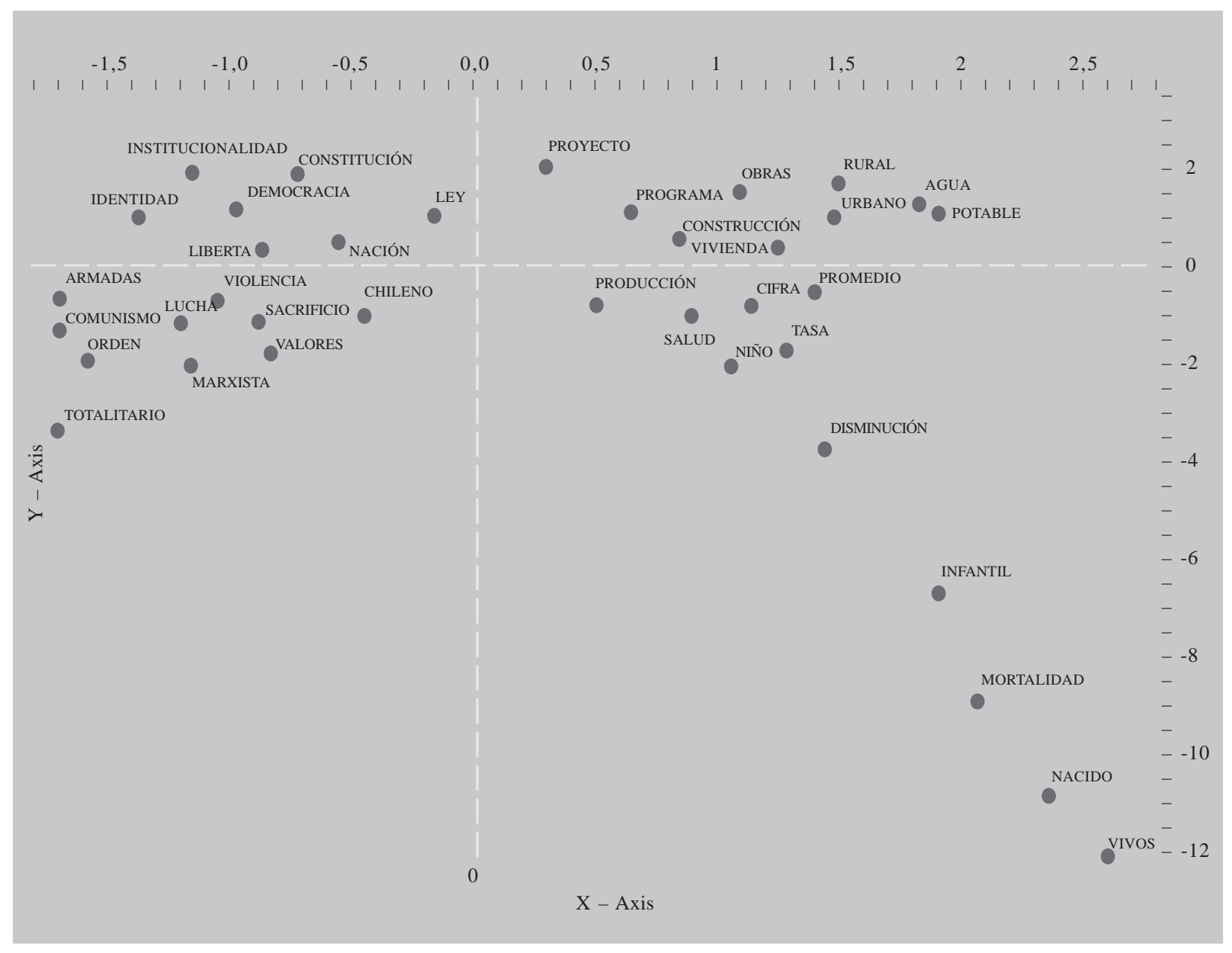

Fuente: Elaborado por los autores.

El primer cuadrante, de gran impacto en la política social neoliberal impulsada por la dictadura, es la que devela su relación con lo mercado-económico. La política neoliberal que Pinochet promueve en su discurso está centralmente vinculada a la noción de desarrollo, idea que está relacionada con un crecimiento de sectores económicos de la vida nacional, con énfasis en la actividad de privados, dejando para los sectores más pobres un área de subsidios para satisfacer necesidades básicas, y la marginal participación en los procesos de capitalización de las empresas públicas mediante el llamado "capitalismo popular" (que implico la liquidación de un cuantioso patrimonio fiscal que pasó a manos de empresas privadas).

Hemos propuesto la etiqueta "mercado", pues el discurso de Pinochet sostenidamente impulsó en sus mensajes la adopción del modelo de mercado en la organización económica del país. Por tanto, no se trata sólo del ámbito económico puramente, sino de un incisivo direccionamiento hacia los postulados de esta corriente de la economía política, clave en la instauración del neoliberalismo, reemplazando el modelo anterior basado en una industrialización nacional sustitutiva de importaciones:

El crecimiento que estimularemos para la economía nacional podrá alcanzarse con un esfuerzo importante de ahorro interno, que deberá ser superior a su tasa histórica. Asimismo, el uso del crédito externo será orientado principalmente al financiamiento de proyectos que promuevan las exportaciones y la sustitución de importaciones.

(A. Pinochet, Mensaje Presidencial, 1983).

En cuanto a la generación de políticas sociales, los discursos de la dictadura están muy vinculados a la evidencia de obras de infraestructura que permitan sostener en el tiempo la "obra" de Pinochet. En 
este sentido, las políticas de agua potable, caminos e inversión en infraestructura estratégica tiene de ser vinculada a los beneficios para los sectores menos favorecidos por los ajustes estructurales impuestos desde la visión neoliberal.

En el sector obras sanitarias, se dio término a la tercera etapa del programa de agua potable rural y de inmediato se inició la cuarta etapa del mismo. Con ello se logrará una cobertura del 82\% de la población rural concentrada, índice que corresponde a uno de los más altos de América Latina.

(A. Pinochet, Mensaje Presidencial, 1986).

En cuanto al segundo sector es el cuadrante de la política social propiamente tal. En el aparecen dos líneas argumentales emergentes. La primera está relacionada con un conjunto de afirmaciones que nos aproximan a un carácter biopolítico de la acción de la dictadura, al enunciar aspectos vinculados con lo demográfico, es decir, con los nacimientos, la mortalidad infantil y las acciones que subsidian la maternidad mediante transferencias económicas a mujeres de sectores de bajos ingresos. En la segunda argumentación relevante, establece que las políticas sociales están relacionadas con el avance del neoliberalismo, cuya retórica ofrece desarrollo-crecimiento al país, peor con los costos derivados del desmantelamiento del Estado Asistencial que rigió hasta 1973:

Se han creado las condiciones socioeconómicas objetivas que permitirán enfrentar el próximo siglo en la condición de un país desarrollado, capaz de superar la pobreza que heredáramos de décadas de estatismo, demagogia y políticas socializantes.

(A. Pinochet, Mensaje Presidencial, 1988).

El tercer cuadrante refiere a los componentes político-ideológicos del discurso de Pinochet, en los que se manifiestan algunas categorías relevantes, como la figura del enemigo, la idealización del orden y la regularidad de la institucionalidad, aspectos claves para la fundamentación de la política social durante la dictadura. El rasgo ideologizante del discurso representa la semántica más abstracta del corpus estudiado, pues remonta a los sustratos conceptuales que sustentan la política social neoliberal de Pinochet en el sometimiento por la fuerza de la población, utilizando además medios jurídicos encapotados y nuevas estrategias comunicativas que abren espacio a la economía de mercado, como oposición al mal representado por el mundo socialista,

La figura del enemigo es encarnada ideológicamente en el marxismo. Existe en el discurso de Pinochet una clara intencionalidad de vincular el concepto de totalitarismo a los grupos de adversarios, que operan en calidad de contradestinatarios, a pesar de que su régimen se presenta cercano a este rasgo. En el discurso, el adversario es catalogado como un peligro asociado a las acciones de terrorismo, que afecta a los más pobres y que condiciona las estrategias de aproximación de la dictadura hacia los sectores más pobres.

En ese lapso el país sufrió la promoción del odio y de la lucha de clases, la acción vil de mercenarios extranjeros que preparaban una guerra civil, el intento de orientar políticamente a la educación, el despojo de la propiedad privada, la politización de sus instituciones y gremios, el desabastecimiento generalizado de bienes y alimentos esenciales y la total postración moral, social y económica. ¡Los políticos marxistas y simpatizantes vestidos de ropajes democráticos y pródigos en declaraciones acerca de la libertad, buscaron sojuzgar a Chile, para luego someterlo a la órbita soviética!

(A. Pinochet, Mensaje Presidencial, 1986).

En el párrafo anterior, tras un intento de regicidio en contra del dictador, es una muestra de la forma en la cual vincula una supuesta guerra civil a eventos ocurridos durante el gobierno de Salvador Allende, amplificando sus consecuencias mediante el manejo de las condiciones de la enunciación. En el fragmento ilustrativo también se utiliza una metáfora para referir a los opositores al régimen, ampliando su base de significación en torno a la figura de los marxistas, categoría en la cual incluye a todos los contradestinatarios del discurso.

En cuanto a la idealización del orden, esta aparece como clave al momento de formular la política social neoliberal de la dictadura. El orden se contrapone al caos al que supuestamente llevó la política pública de la Unidad Popular. Como es posible apreciar en el siguiente fragmento ilustrativo, el progreso del país está de lado de quienes son co-responsables de mantener el orden instaurado por la dictadura, y para eso, se le ofrece a estos sectores la posibilidad de desarrollar sus intereses privados.

El Gobierno ha estado abierto a todos aquellos sectores políticos respetuosos del orden vigente, que quieran participar en el avance institucional, con aportes, sin que ello implique renunciar a sus intereses. 
Consideramos que la tarea de construir una democracia estable para nuestro país no solo es responsabilidad de algunos, sino que de todos los chilenos.

(A.Pinochet, Mensaje Presidencial, 1987).

Un aspecto final en este punto tiene relación con la regularidad institucional, y que actúa como corolario de las dos características antes analizadas. El orden institucional queda asentado a partir de un plebiscito espurio celebrado en septiembre de 1980 que valida la Carta Constitucional maquinada por la estructura política de la dictadura. Esta Constitución contiene los principales lineamientos de derechos de máximo rango en los cuales, se establecen las exclusiones a los enemigos (art. $8^{\circ}$ ) y se indican los pilares del nuevo orden sociopolítico de Chile (Cáp. I de la Constitución). La regularidad constitucional se alcanzará, según el plan trazado por la dictadura, en marzo de 1990, diseño estratégico que establecía una potencial extensión del régimen hasta 1996, situación que fue interrumpida por la decisión popular de negar dicha prolongación, gracias a la derrota que sufre Pinochet en el plebiscito presidencial de 1988.

En el siguiente fragmento seleccionado, es posible advertir la forma en que el dictador alude al avance de la nueva institucionalidad. La regularización del orden excluye a los enemigos, utilizando en ella la retórica de los derechos humanos que su mismo régimen violaba sistemáticamente desde 1973. En este fragmento, Pinochet también centra en la libertad como dimensión central del nuevo orden basado en el neoliberalismo, el cual está plenamente reflejado en el art. 19 de la Constitución Política de Chile. Esta sección del cuerpo constitucional entroniza la libertad de consumo y la libertad de los privados para introducirse en áreas de la sociedad que hasta el momento estaban reservados para la acción del Estado. Un ejemplo de ello, y cuyas consecuencias se extienden hasta nuestros días, tiene relación a la libertad de enseñanza, con la consecuente apertura de proyectos comerciales asociados a la educación superior universitaria.

Al promover un progresivo avance del país hacia la consolidación de una nueva institucionalidad, lo hacemos considerando que la mayor amenaza para los derechos humanos proviene de la tiranía totalitaria y de la violencia terrorista, concepción que se reafirma en nuestra Constitución, la que es categórica para excluir de la vida cívica toda doctrina que atente contra nuestra libertad. El texto constitucional contiene, además, una clara definición frente al terrorismo, al cual considera por esencia contrario a los derechos humanos y expresión de cobardía y crueldad.

(A. Pinochet, Mensaje Presidencial, 1981).

El último de los cuadrantes es el ideológico que institucionaliza las consecuencias de la adopción del neoliberalismo por parte del régimen de Pinochet. En este sector confluyen un conjunto de términos asociados a la regularización jurídica-constitucional de la dictadura, ámbito que da un soporte institucional necesario para el cambio del modelo socioeconómico de desarrollo, e impulsa la adscripción a un nuevo orden, en el cual, el Estado de Chile se escinde de sus patrimonios materiales y simbólicos más relevantes: de este modo, se da paso a un sustrato ideológico sobre el cual se sostienen las políticas sociales de la dictadura.

Pero también, y en forma muy importante, se incorporó a los propios trabajadores a los beneficios de una economía social de mercado, justa y solidaria, por la vía de convertirlos en copropietarios de las respectivas empresas.

(A.Pinochet, Mensaje Presidencial, 1989).

\section{Conclusiones}

En relación al método empleado, éste nos permite reducir la complejidad de un conjunto de textos que abarca un periodo de nueve discursos que suman 80.821 palabras, 2.061 párrafos, lo cual contribuye a generar una visión extensa de la construcción de los discursos políticos vinculada al neoliberalismo y su impronta en la política social.

Los principales ejes de la política social de Pinochet están impregnados de los contenidos neoliberales, que al momento de pronunciar los Mensajes Presidenciales analizados (1981-1989), regía como el modelo imperante en la vida social chilena post golpe de Estado. Una mirada de segundo orden nos permite indicar que en los discursos analizados se cruzan las declaraciones de política social de salud, educación, vivienda e ingresos. Ocurren dos procesos más bien complementarios: por una parte se declara abiertamente el contraste de la política social supuestamente modernizante de la dictadura con un pasado de énfasis estatal, que en la lectura de la dictadura, sólo trajo postergaciones y retrasos sociales. Para eso, el dictador pretende representarle a los 
contradestinatarios (opositores, enemigos) los logros, avances en materia de política social en las áreas antes descritas. Los discursos están en este sentido, sobrecargados de cifras e intentos de evidenciar un supuesto avance en los diversos campos sociales, azuzando además una amenaza explícita a los grupos oponentes.

Por otro lado, la política social es parte de una estrategia discursiva demostrativa, que busca también profundizar la vinculación con los partidarios de la dictadura (prodestinatarios), atendiendo además todas las evidencias para conquistar la voluntad de los indecisos (paradestinatarios). Como supuesto, la política social está al servicio de las cifras del gobierno dictatorial y no de los grupos humanos más desfavorecidos. Esto queda reforzado con la mínima expresión de la participación como componente de estos discursos.

El uso de la política social como "arma” ideológica, puede resultar clave para comprender los procesos y resultados finales de cuarenta años de neoliberalismo en Chile, una vez terminada formalmente la dictadura en 1990, pero incólume en sus sentidos centrales durante la seguidilla de gobiernos que sucedieron durante la llamada "Transición Democrática". Es en la actualidad de las prácticas actuales de gubernamentalidad que asientan, por ejemplo, la privatización de la educación y la previsión en Chile, la que ha motivado este estudio. Esto implica reconocer en los discursos emitidos cada 11 de septiembre entre 1981 y 1989 , la posibilidad de encontrar en la altisonancia de la dictadura algunas pistas sobre la ruta de la actual política social neoliberal chilena.

\section{Referencias}

CARBALLO, A. et al. La historia oculta del régimen militar. Santiago de Chile: La Época, 1990.

ELSTER, J. Reflexiones sobre marxismo, funcionalismo y teoría de juegos. En: ROEMER, J. E. (Org.). El marxismo: una perspectiva analítica. México: Fondo de Cultura Económica, 1989.

FAIRCLOUGH, N. Critical Discourse Analysis. Nueva York: Longman, 1995.

FAIRCLOUGH, N. El Análisis Crítico del Discurso como método para la investigación en ciencias sociales. En: WODAK, R.; MEYER, M. (Ed.). Métodos de análisis crítico del discurso. Barcelona: Gedisa, 2003.

FOUCAULT, M. Nacimiento de la biopolítica. Buenos Aires: Fondo de Cultura Económica, 2012.

GERSTLÉ, J. La comunicación política. Santiago de Chile: LOM, 2005.

ILLANES, M. A. En el nombre del pueblo, del Estado y de la ciencia. Santiago de Chile: Ministerio de Salud, 2010.

LARRAÍN, J. El concepto de ideología. Postestructuralismo, postmodernismo y postmarxismo. Santiago de Chile: LOM, 2010. (v. 4). SOLLON, 2003. Acción y texto: para una comprensión conjunta del lugar del texto en la (inter) acción social, el análisis mediato del discurso y el problema de la acción social. En: WODAK, R.; MEYER, M. (Ed.). Métodos de análisis crítico del discurso. Barcelona: Gedisa, 2003.

VAN DIJK, T. A. La ciencia del texto: un enfoque interdisciplinario. Barcelona: Paidós, 1992.

Ideología y discurso. Barcelona: Ariel, 2003.

Estructuras y funciones del discurso: una introducción interdisciplinaria a la lingüística del texto y a los estudios del discurso.

México: Siglo XXI, 2007.

VERÓN, E. La palabra adversativa. Observaciones sobre la enunciación política. En: VERÓN, E. et al. El discurso político. Lenguajes y acontecimientos. Buenos Aires: Hachette, 1987.

WODAK, R. De qué trata el análisis crítico del discurso (ACD). Resumen de su historia, sus conceptos fundamentales y sus desarrollos. En: WODAK, R.; MEYER, M. (Ed.). Métodos de análisis crítico del discurso. Barcelona: Gedisa, 2003.

\section{Juan Saavedra Vásquez}

jsaavedr@ubiobio.cl

Trabajador Social

Doctor en Ciencias Humanas

Académico del Departamento de Ciencias Sociales de la Universidad del Bío-Bío

\section{Fernando Farías Olavarría}

ffarias@ubiobio.cl

Trabajador social

Magíster en Investigación Social y Desarrollo

Académico del Departamento de Ciencias Sociales de la Universidad del Bío-Bío 


\section{Universidad del Bío-Bío}

Escuela de Trabajo Social

Avda. Collao 1202

Casilla 5-C

CP: 4050231

Concepción - Chile 